\title{
BMJ Open Enhanced Recovery After Surgery implementation in practice: an ethnographic study of services for hip and knee replacement
}

\author{
Sarah Drew, ${ }^{1}$ Andrew Judge, ${ }^{1}$ Rachel Cohen, ${ }^{2}$ Raymond Fitzpatrick, ${ }^{3}$ \\ Karen Barker, ${ }^{4}$ Rachael Gooberman-Hill ${ }^{1}$
}

To cite: Drew S, Judge A, Cohen $\mathrm{R}$, et al. Enhanced Recovery After Surgery implementation in practice: an ethnographic study of services for hip and knee replacement. BMJ Open 2019;9:e024431. doi:10.1136/ bmjopen-2018-024431

- Prepublication history and additional material for this paper are available online. To view these files, please visit the journal online (http://dx.doi. org/10.1136/bmjopen-2018024431).

Received 25 May 2018 Revised 12 November 2018 Accepted 12 December 2018

Check for updates

(C) Author(s) (or their employer(s)) 2019. Re-use permitted under CC BY-NC. No commercial re-use. See rights and permissions. Published by BMJ.

${ }^{1}$ Musculoskeletal Research Unit, Translational Health Sciences, Bristol Medical School, University of Bristol, Bristol, UK ${ }^{2}$ School of Population Health Sciences, Centre for Academic Mental Health, University of

Bristol, Bristol, UK

${ }^{3}$ Nuffield Department of

Population Health, University of Oxford, Oxford, UK

${ }^{4}$ Nuffield Department of

Orthopaedics, Rheumatology and Musculoskeletal Sciences, University of Oxford, 0xford, UK

Correspondence to

Sarah Drew;

sarah.drew@bristol.ac.uk

\section{ABSTRACT}

Objectives Enhanced Recovery After Surgery (ERAS) programmes aim to improve care quality by optimising components of the care pathway and programmes for hip and knee replacement exist across the UK. However, there is variation in delivery and outcomes. This study aims to understand processes that influence implementation using the Consolidated Framework for Implementation Research (CFIR) to inform the design and delivery of services.

Design An ethnographic study using observations and interviews with staff involved in service delivery. Data were analysed using a thematic analysis, followed by an abductive approach whereby themes were mapped onto the 31 constructs and 5 domains of the CFIR.

Setting Four hospital sites in the UK delivering ERAS services for hip and knee replacement.

Participants 38 staff participated including orthopaedic surgeons, nurses and physiotherapists.

Results Results showed 17 CFIR constructs influenced implementation in all five domains. Within 'intervention characteristics', participants thought ERAS afforded advantages over alternative solutions and guidance was adaptable. In the 'outer setting', it was felt ERAS should be tailored to patients and education used to empower them in their recovery. However, there were concerns about postdischarge support and tensions with primary care. Within the 'inner setting', effective multidisciplinary collaboration was achieved by transferring knowledge about patients along the care pathway and multidisciplinary working practices. ERAS was viewed as a 'message' that had to be communicated consistently. There were concerns about resources and high volumes of patients. Staff access to information varied. At the domain 'characteristics of individuals', knowledge and beliefs impacted on implementation. Within 'process', involving opinion leaders in development and 'champions' who acted as a central point of contact, helped to engage staff. Formal and informal feedback helped to develop services.

Conclusions Findings demonstrate successful implementation involves empowering patients to work towards recovery, providing postdischarge support and promoting successful multidisciplinary team working.
Strengths and limitations of this study

- This study contributes to an understanding of how Enhanced Recovery After Surgery services for hip and knee replacement can be effectively implemented.

- Using ethnography that combined interviews and observations provided a rounded, in-depth understanding of practice.

- Including 38 professionals from four study sites provides confidence that findings are transferable to other settings.

- Including different numbers of participants from study sites meant some hospitals could have been over-represented in the analysis but this was mitigated by analysing data from each hospital as a discrete data set.

- Conducting a thematic analysis and transposing themes onto the theory that was considered the best 'fit' for the data ensured data were not forced into predefined constructs.

\section{BACKGROUND}

Over 4.7 million patients are admitted for surgery in the UK each year. ${ }^{1}$ To reduce the length of stay, lessen readmission rates and improve care quality, Enhanced Recovery After Surgery (ERAS) programmes have been introduced across the healthcare system. ERAS programmes aim to optimise preoperative, perioperative and postoperative care in a range of surgical specialties. Programmes may use minimally invasive surgical techniques, effective pain management, early postoperative mobilisation, comprehensive patient education and discharge plans that have been tailored to meet individual patient needs. ERAS involves a close collaboration between healthcare professionals and patients who are invited to work towards their own recovery and rehabilitation. ${ }^{2}$

An ERAS programme for hip and knee replacement was introduced with support 
from the Department of Health in 2009. ${ }^{3}$ Around 160000 total hip and knee replacement surgeries are carried out each year in England and Wales, ${ }^{4}$ usually to relieve pain and improve function for people with osteoarthritis. Joint replacement involves removing part of a joint and replacing it with a prosthesis. ${ }^{5}$ This is a major operation that has significant benefits, but also known risks and adverse outcomes. ${ }^{6}{ }^{7}$ Research has demonstrated that ERAS orthopaedic pathways reduce the length of stay and mortality rates. ${ }^{89}$ However, there is significant variation in how these programmes are delivered, ${ }^{10}$ along with variation in health outcomes. ${ }^{11}$ Reasons for this are unclear.

A recent systematic review of existing qualitative studies exploring staff experiences of delivering ERAS identified a number of factors that impacted on successful implementation. These included communication and collaboration between staff, attitudes to change, the use of clinical protocols to standardise care, expectations around the intervention and the embedding of ERAS into everyday practice ${ }^{12}$ However, no studies have explored factors that impact on the implementation of ERAS programmes for hip and knee replacements. Since these involve a considerable postdischarge commitment from patients, experiences of service implementation may differ from other conditions. It is unclear which factors identified in previous research may be transferable to this context.

Theoretical frameworks are increasingly used to understand the implementation of complex interventions such as ERAS and these help to provide a more comprehensive explanation of how and why interventions can be successfully implemented in practice. ${ }^{13}$ The Consolidated Framework for Implementation Research (CFIR) outlines 31 constructs that impact on processes of implementation, grouped into five domains. These are: (1) intervention characteristics that relate to the attributes of an intervention; (2) outer setting or external influences; (3) inner setting or factors within an organisation; (4) characteristics of individuals that are the behaviours of individuals tasked with enacting the intervention and (5) process that is the planning and delivery of an intervention. ${ }^{14}$ Unlike other theories and frameworks that derive from the field of implementation science, the CFIR focuses on the importance of meeting patient needs in service design. CFIR has been successfully used to explore the implementation of a range of healthcare interventions. ${ }^{15-17}$

This study aims to understand the organisational processes that help or hinder the implementation of ERAS programmes for hip and knee replacement. Doing so will provide information about how best to organise and deliver these services to provide effective patient care.

\section{METHODOLOGY}

This is an ethnographic study that involved spending extended periods of time collecting qualitative data at study sites to generate in-depth understandings. ${ }^{18}$ Ethnography is a methodology that involves immersing oneself in a setting for an extended period of time to help understand social systems from the perspective of its inhabitants. As such, it provided an ideal means of exploring contextual factors that impacted on the implementation of services. ${ }^{19}$ This forms part of a broader study that includes patient experiences of accessing services. Data collection was undertaken between November 2016 and March 2017 and carried out by one of the research team members involved in planning and carrying out the study (RC). The researcher was employed by the University of Bristol and unknown to study participants.

\section{Hospital sites}

Maximum variation sampling was used to identify four hospitals from England with a range of characteris$\operatorname{tics}^{20}$ : a teaching hospital, a district general hospital, a specialist orthopaedic hospital and an independent sector treatment centre. This was intended to capture experiences in a range of contexts.

\section{Observation sessions and job shadowing}

Potential participants were identified by a staff member working in orthopaedics with knowledge of staffing at each site. Potential participants were then sent a study information pack that included information about the study, invitation letter and reply slip to return if they were interested in participating. Snowball sampling was also used such that participants recommended other potential participants. $^{21}$

Observation sessions were conducted at each study site. To inform data collection, an observation checklist was devised with input from the multidisciplinary research team and used to explore the clinical setting, activities taking place, treatment protocols and factors that impacted on the implementation of services (see online supplementary file 1 ). The observation checklist was intended to be used flexibly to guide data collection and enable follow-up on issues considered relevant to the study. Informal interviews were also used. Data were written up into full field notes. To inform further data collection, memos or reflective notes were used to record emerging ideas. A total of 19 staff agreed to be shadowed and approximately 160 hours of fieldwork were conducted Monday to Friday during working hours ( 5 days of approximately 8 hours at each study site).

\section{Semistructured interviews}

Face-to-face semistructured interviews were undertaken with healthcare professionals involved in service delivery. Interviews were between around 30 and $60 \mathrm{~min}$ long. Thirty-one healthcare professionals participated in interviews, of whom 12 had also participated in observations. A 'topic guide' or list of themes to explore in the interviews was devised based on data collected during observation sessions (see online supplementary file 2). Interviews focused on participants' views and experiences 
of delivering ERAS and factors impacting on implementation. The topic guide was not structured around the constructs of CFIR since we wanted to ensure that the experiences of participants were not 'forced' into predefined concepts. The topic guide was flexible to enable follow-up on issues raised. Interviews were audiorecorded, transcribed and anonymised.

\section{Analysis}

Analysis was iterative and ongoing and informed further data collection. Analysis was carried out in two phases which involved an interim and final phase. Written field notes and transcripts of interviews were anonymised and imported into NVivo software for analysis. Interview transcripts and field notes were analysed using an inductive thematic approach ${ }^{22}$ to identify themes and subthemes in the data. On account of the variation in service delivery between sites, data from each hospital site were analysed as a discrete dataset. Twenty per cent of transcripts were double coded by another member of the research team (RG-H). Codes were then discussed and refined to reach a single code list. As part of the interim analyses, the CFIR was identified as a means of further structuring analysis because of its focus on the importance of meeting patient needs in service design. ${ }^{23}$ Using the CFIR as part of analysis involved transposing themes that had been coded inductively onto the 31 constructs of the framework, grouped into the five domains: (1) 'intervention characteristics', (2) 'outer setting', (3) 'inner setting', (4) 'characteristics of individuals' and (5) 'process'. This was an 'abductive' approach to analysis which involves adopting an existing hypothesis or theory that forms the basis of further investigation. The approach emphasises the importance of collecting detailed fieldnotes, constant comparison of data, memo writing to help develop theoretical categories and the searching of negative cases to test the theory. This enabled us to apply existing theory while ensuring data were not forced into predefined constructs, as described by Tavory and Timmermans. ${ }^{24}$ Interpretive accounts of the data were then generated.

Informed consent from participants was collected prior to data collection. This confirmed that participants understood participation was voluntary and that they were willing to let the researcher (SD) use anonymous quotations from them in the write up of the study. Each hospital site provided Research and Development approval.

\section{Patient and public involvement}

To refine the study design and data collection materials, we collaborated with members of 'The Patient Experience Partnership in Research' (PEP-R) group. PEP-R is the dedicated patient involvement group based in the Musculoskeletal Research Unit, University of Bristol. PEP-R comprises patients with musculoskeletal conditions, many of whom have had a joint replacement. ${ }^{25}$ A written overview of study findings will be provided to participants once the study, of which this qualitative work forms a part, has been completed.

\section{Findings}

Characteristics of ERAS services for hip and knee replacement are displayed in figure 1 . These present summarised information only to preserve the anonymity of sites.

\section{Sample characteristics}

The 38 participants included 10 physiotherapists or occupational therapists, 18 nurses, 5 orthopaedic surgeons, 1 anaesthetist, 1 matron, 2 therapy technician assistants and 1 theatre manager. Twelve staff participated in interviews and observations, 19 took part in interviews only and 7 in observations only. Between 4 and 14 participants took part from each study site. Participants' characteristics are displayed in table 1, which presents summarised information by study site to avoid the potential for identification of individual participants. We use pseudonyms for study sites.

A total of 17 CFIR constructs were seen to influence the processes of implementation for ERAS programmes in all five domains of the framework. A summary of the themes identified and their relationship to these constructs and domains are outlined in table 2.

Below we explore factors that impact on the implementation of ERAS services using the five domains of CFIR in more detail. See box 1 for illustrative quotes for each domain.

\section{Intervention characteristics}

Participants expressed enthusiasm for the relative advantages of ERAS since the shortened length of stay had resource and cost-saving implications. There was a sense that ERAS involved a compromise between reducing the length of stay while ensuring this did not lead to an increase in readmission and complication rates. One participant described this as a 'trade-off'. At the district general hospital (Shinebury), care pathways were developed internally by consultant surgeons who piloted the intervention and communicated findings to staff. This helped generate internal support. By contrast, a nurse sister at the specialist orthopaedic hospital (Elmfield) described how ERAS practices had been 'introduced on us' and suggested that having someone to lead its development would have inspired enthusiasm.

ERAS was seen to be adaptable and this was viewed as a strength since it could be modified to work within individual hospital contexts. None of those interviewed had seen any formal policies issued by the Department of Health, although many were aware it was a government initiative. There was variation in opinion on which patients should be included in the pathway. At the district general hospital (Shinebury), all patients were included whereas at the specialist orthopaedic hospital (Elmfield), patients were only selected if it was felt they were healthy enough for rapid discharge. How ERAS was understood 
Towerton

Year ERAS first introduced: 2011

Key features include:

- $\quad$ ERAS nurse champion as central coordinator

- Patients provided with five separate leaflets including information about anaesthetic, monitoring wounds, blood clots

- Patients attend hip or knee school for further information

- $\quad$ Pre-assessment includes medical history and consideration of social history and current living arrangements

- Physiotherapists see patients a minimum of one day after surgery

- Links with other locality services for post discharge support put in place before admission. 'Step-down' ward used for patients who aren't well enough to go home.

- Post-operative follow up appointments conducted at six weeks, six months and 12 months.

- Hip and knee patients follow same care pathway.

- Only patients that have attended a hip or knee school are classed as "ERAS patients".

\section{Lastmere}

Year ERAS first introduced: 2014/ 2015

Key features include:

- No designated coordinator but informally, physiotherapists act as key points of contact.

- $\quad$ Patients provided with one comprehensive hip or knee 'joint guide' booklet with information about hospital, surgical procedures and rehabilitation including safety and exercise.

- $\quad$ Patients attend hip or knee school that includes information on exercises, practice with mobility aids and advice on likely length of stay.

- Pre-operatively, patients complete questionnaires about their home environments so their rehabilitation needs can be assessed.

- Patients are seen by physiotherapists post-operatively, preferably on the same day of surgery or day one at the latest. Patients are seen twice a day.

- $\quad$ Post-operative follow up appointments conducted at six weeks by consultants. Patients also seen by physiotherapy team at least three or four times during the first six weeks, either by attending rehabilitation classes or in one to one appointments. All patients are seen within the first two weeks after surgery.

- Hip and knee patients follow same care pathway.

- All patients are included in the ERAS care pathway.
Shinebury

Year ERAS first introduced: Piloted in 2000

Key features include:

- No central coordinator

- Patients provided with leaflets and DVDs for information. DVD includes information about surgery, previous patients talking about their experiences.

- $\quad$ Patients attend hip or knee school, including demonstration of rehabilitation exercises

- Pre-assessment involves full medical checks. Patients see occupational therapists and complete questionnaires about their home environments to assess level of support needed post discharge.

- $\quad$ Patients seen by physiotherapists and mobilised day one after surgery

- Limited organisational support available for patients post discharge and formal care packages are difficult to put in place.

- $\quad$ Post-operative follow up appointments conducted at two weeks, six weeks and one year, run by the lead physiotherapist, occupational therapists and specialised orthopaedic nurses. Follow up also takes place in fracture clinics at five and ten years.

- Hip and knee patients follow same care pathway.

- $\quad$ All patients are included in the ERAS care pathway.

Woodland

Year ERAS first introduced: Around 2010

Key features include:

- No central coordinator

- Referrals from primary care are through a musculoskeletal 'hub', a triage system run by consultants to ensure patients receive the correct treatment

- Patients having hip surgery attend a 'school'. No school exists for those undergoing knee replacement

- There is a same day assessment clinic, a "one-stop shop" which involves a full pre-operative assessment, including full observations and occupational therapy assessment. Only hip school patients see physiotherapists.

- All discharge planning is done by physiotherapists.

- $\quad$ Post-operative follow up appointments conducted at six weeks by consultants.

- Hip and knee patients do not follow the same care pathway.

- Not all patients are assigned to the ERAS care pathway for hip surgery and this is done at the discretion of consultants. Those considered to be more frail or complex are less likely to be included in ERAS.

Figure 1 Characteristics of ERAS services for hip and knee replacement at four study sites. ERAS, Enhanced Recovery After Surgery. 
Table 1 Participant characteristics (aggregated to ensure anonymity)

\begin{tabular}{lll}
\hline Type of centre with pseudonym & Profession & Time spent in role at site \\
\hline District general hospital & 2 physiotherapists & 5 to 14years \\
Shinebury & 7 staff nurse/sisters & 2 weeks to 11 years \\
& 3 consultant orthopaedic surgeons & 4 years to 21 years \\
& Consultant anaesthetist & 22 years \\
Specialist orthopaedic hospital & 2 physiotherapists & 3 years- to 15years \\
Elmfield & 3 occupational therapists & 18 months to 12years \\
& 6 staff nurse/sister/nurse specialists & 1 month to 1 year \\
& Matron & 1 month \\
Teaching hospital & 1 consultant orthopaedic surgeon & 10 years \\
Towerton & 1 physiotherapist & 10 years \\
& 2 therapy technician assistants & 1 to 2years \\
& 4 staff nurse/sister/nurse specialists & 3 months to 7 years \\
Independent sector treatment centre & 2 physiotherapists & 3 years \\
Lastmere & 1 staff nurse & 2 to 4years \\
\hline
\end{tabular}

and implemented in practice, therefore, differed across sites.

\section{Outer setting}

Participants viewed ERAS as a 'partnership' between staff and patients. As a result, meeting patient needs was viewed as being essential to ensuring it worked effectively. A number of patients could not be discharged because they were medically unfit but there were also patients seen to be resistant to rapid discharge. To address these issues, a nurse at the specialist orthopaedic hospital (Elmfield) thought it was crucial to adapt approaches to suit individual needs, adopting a recovery time that was manageable. Another emphasised the importance of education as a way of 'breaking down' attitudes that acted as a barrier to discharge.

Purposes of patient education were considered manifold. The most important was to attribute agency to patients to give them ownership of their recovery. Reported benefits were that patients were easier to manage postoperatively as they knew what to expect which impacted positively on recovery trajectories. Information was distributed in a range of formats. Written information helped to reinforce information provided at consultation and gave patients a source to refer back to. Sites operated hip and knee schools, regular classes designed to educate patients about their treatment and participants thought that the 'group dynamic' created a safe space for asking questions and sharing experiences. Face-to-face contact was seen as an opportunity to clarify information.

Participants conceptualised ERAS as a 'message', which had to be consistently communicated so that patients understood and adhered to aspects of care. It was thought that if this was 'diluted', then understanding and adherence could be reduced. Participants thought that new staff who were not familiar with ERAS and those who were 'not buying into the process' might provide inaccurate information. This is discussed in further detail below.

Participants from all sites were concerned about postdischarge support. According to a nurse at the specialist orthopaedic hospital (Elmfield), this was important as patients 'panicked' without it. There was variation in postdischarge services provided by the sites. One participant felt that providing a telephone number as a point of contact was 'the absolute minimum'. Staff thought this made patients feel more 'secure'.

'Cosmopolitanism' or cooperation with external agencies was important since successful implementation depended on how effectively services worked with practitioners in primary care at the point of referral and discharge. Staff suggested general practitioners (GPs) did not always understand the practicalities of ERAS which manifested itself in inappropriate referrals or giving patients unrealistic expectations about potential outcomes.

Participants highlighted some gaps in communication between primary and secondary care after patients' discharge. One felt that GPs sometimes questioned patients' readiness to return home, another that they got 'cross' when they thought patients had been discharged without sufficient pain relief. Participants at the district general hospital (Shinebury) and the specialist orthopaedic hospital (Elmfield) were also worried that patients had no point of contact if they were experiencing difficulties before their follow-up review, meaning they had to return to primary care, placing an unnecessary burden on services. Furthermore, there were concerns that GPs 


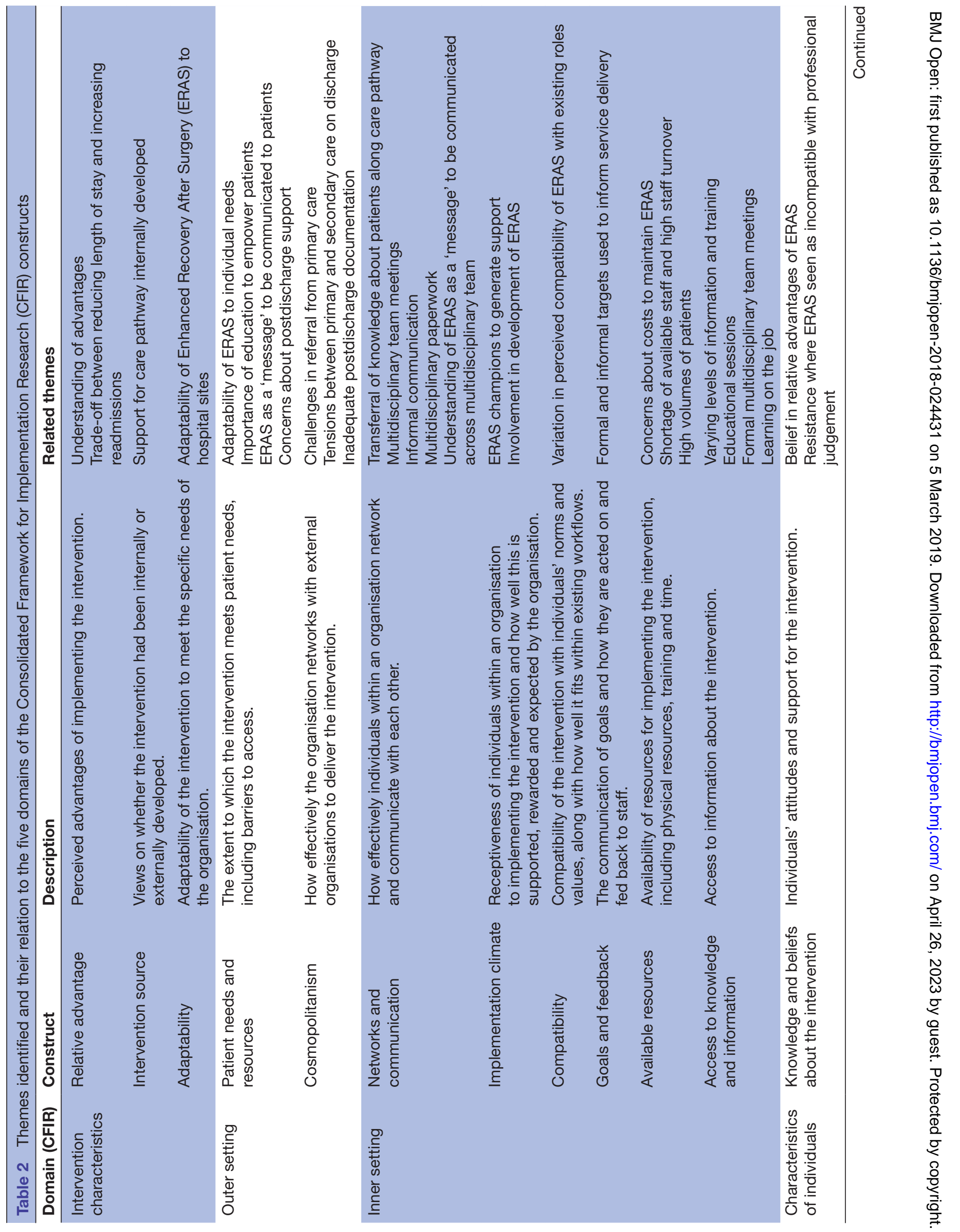




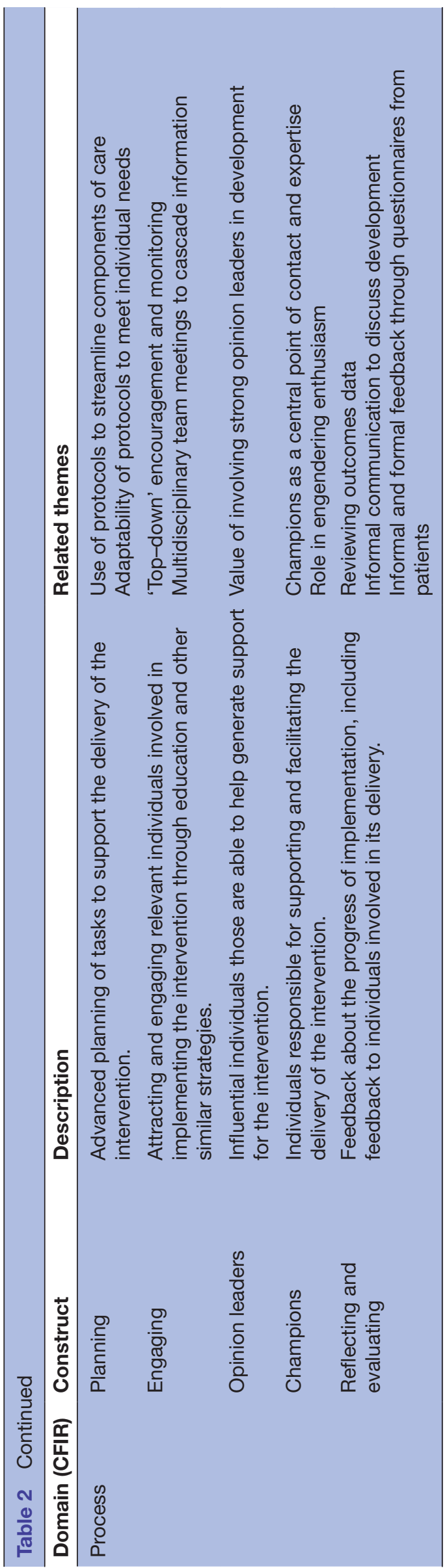

were not provided with adequate postdischarge documentation. According to a nurse at the specialist orthopaedic hospital (Elmfield), there was a need to further engage with GPs and community services.

\section{Inner setting}

It was felt that one of the key elements of success was effective networking and communication between staff. Multidisciplinary team members tended to operate in 'silos' with responsibility for delivering different components of care. To communicate patient information, knowledge had to be transferred along the care pathway as part of a 'logical progression'. However, a nurse at the teaching hospital (Towerton) was concerned that those undertaking preassessment were not consistently transferring information, meaning that potentially important details were missed.

Regular multidisciplinary team meetings to discuss ERAS were advocated although these were challenging to organise. Informal communication was seen as being important and the location of staff in close proximity was seen to facilitate this. Nurses, physiotherapists and occupational therapists at the district general hospital (Shinebury) and the independent sector treatment centre (Lastmere) ran 'joint clinics' together and doing so encouraged collaboration. Multidisciplinary documentation was also valued although the quality and consistency of this varied. For instance, at the specialist orthopaedic hospital (Elmfield), paperwork did not identify whether patients had been assigned to the ERAS pathway. This reflects norms of practice in a busy communicative context. A physiotherapist at the independent sector treatment centre (Lastmere) viewed documentation as a 'back-up' since staff were in 'constant communication' with one another.

A number of participants characterised ERAS as a 'message' that needed to be communicated across the multidisciplinary team to ensure that its components were being consistently delivered. However, this was not always achieved. For instance, at the specialist orthopaedic hospital (Elmfield), surgeons did not always agree with one another about which patients were eligible for ERAS. ERAS champions helped to ensure that the 'message' was successfully communicated and that staff were delivering components of care consistently.

Regarding the implementation climate, participants described the importance of a collective ethos and 'belief' in ERAS. ERAS champions helped to garner support from the multidisciplinary team. However, at the specialist orthopaedic hospital (Elmfield), there seemed to be no clear leadership. Furthermore, not all team members were invited to meetings to discuss ERAS development and this made them feel less engaged.

There was variation in how compatible ERAS was seen to be with existing roles. A number of participants thought that ERAS involved expanding on existing working practices, making it easy for them to do the necessary work. However, some anaesthetists were reportedly resistant as 
they preferred using their own professional judgement to following protocols. Similarly, a participant at the teaching hospital (Towerton) had found it difficult to change nursing practice as colleagues were uncomfortable about encouraging patients to be so independent and were reluctant to send them home. These challenges existed across all study sites.

Targets and goals for the length of stay were established formally by the Hospital or Trusts and informally by ERAS services. Performance against formal and informal targets was fed back to staff and used as a basis to collaboratively improve service delivery. Failure to meet the formal length of stay targets led to fines meaning staff felt under substantial pressure to meet these goals.

The financial cost of maintaining ERAS was a concern, although the extent and nature of this varied. At the specialist orthopaedic hospital (Elmfield), staff were particularly worried about lack of current funding that meant they were not able to acquire sufficient staff or facilities such as beds. Staff at the independent sector treatment centre (Lastmere) thought funding cuts may prevent them from providing patient information booklets that they saw as being central to effective rehabilitation.

A shortage of available staff and high staff turnover were seen as creating difficulties as it meant colleagues had to do additional work and struggled to find time to deliver care. At the district general hospital (Shinebury), time constraints were seen to make it more difficult to arrange formal care packages after discharge and impacted on the quality of postoperative follow-up. A deputy nurse sister at the specialist orthopaedic hospital (Elmfield) thought that follow-up reviews should be undertaken by nurses or physiotherapists, as they were at the district general hospital (Shinebury), to relieve the 'pressure' on consultants. High volumes of patients at the district general hospital (Shinebury) and the teaching hospital (Towerton) were also seen to place pressures on services. Since major trauma was prioritised over elective surgery, operations were often cancelled at short notice. To relieve the burden on services, responsibilities for ERAS had been shifted across existing and new staff roles.

Staff at the four sites had received varying levels of access to knowledge and information about ERAS. The teaching hospital (Towerton) appeared to have the most comprehensive training and education and staff spent time with the nurse champion who ran educational sessions and incorporated information on ERAS into ongoing orthopaedic training. Staff at the district general hospital (Shinebury) were also expected to attend joint school to help them to educate patients more effectively. By contrast, a participant at the specialist orthopaedic hospital (Elmfield) explained how the intervention had been introduced without any formal education and that this had not been effective. Multidisciplinary team meetings were used as a way of communicating information about changes in working practice, along with 'learning on the job'.

\section{Box 1 Illustrative quotes}

\section{Intervention characteristics}

[ERAS was] revolutionary... especially for the older nurses who had been there 20 years [Senior Sister, teaching hospital (Towerton)]

When you've seen a patient with enhanced recovery protocols, you never want to go back to how you did things before... [seeing how quickly patients recover] was just an amazing transformation. [Consultant surgeon, teaching hospital (Towerton)]

\section{Outer setting}

You've got to bring the patient on board too. You've got to persuade them to go with the flow. [Consultant surgeon, district general hospital (Shinebury)]

You're the one who's going to make [the joint] work, so let's get you working it. This is yours. It doesn't belong to the NHS. It doesn't belong to the surgeon. This is yours'. [It's about giving] them the ownership and the responsibility. [Deputy Sister, specialist orthopaedic hospital (Elmfield)]

\section{Inner setting}

We [the physiotherapists] can actually gather information to save going through things... [the occupational therapist] might have gathered something that perhaps I might take an hour to get out of somebody. [Physiotherapist, district general hospital (Shinebury)]

The other thing that will sometimes get in the way is if the [ERAS] message has been diluted at some point [Consultant surgeon, district general hospital (Shinebury)]

I think there are other people that have the same beliefs as my beliefs... the bond, the desire [to implement ERAS] is uniform from top to bottom. [Consultant Surgeon, teaching hospital (Towerton)]

The sadness we have is we did have a fabulous all singing and dancing booklet but it was funded by a particular company [who is no longer providing support]... the funding for that isn't possible [anymore]. [Physiotherapist, specialist orthopaedic hospital (Elmfield)]

Giving [patients] enough time to ask questions I think is important so it's about having an appropriate length of clinic appointments which obviously [presents] a conflict between seeing a number of patients that the Trust wants you to but giving patients enough time to do that. [Consultant surgeon, specialist orthopaedic hospital (Elmfield)]

Having 'enough capacities for the key professionals to interact with the patient at the right time, from pre-op to post-op [is difficult] [Consultant surgeon, teaching hospital (Towerton)]

\section{Characteristics of individuals}

Every anaesthetist was just doing his own individual recipe and it was very difficult... [it] took quite a lot of engagement to get the anaesthetists to really champion it and get their colleagues to embrace that. [Occupational therapist, specialist orthopaedic hospital (Elmfield)] 


\section{Box 1 Continued}

\section{Process}

The idea [of the meetings] was to keep reviewing the figures and make sure there was an emphasis that everybody cascade to their own colleagues about how we were doing and whether we [were] dropping off on our Rapid Recovery... it's been a challenge to keep that momentum going. [Consultant surgeon, district general hospital (Shinebury)]

\section{Characteristics of individuals}

Within this domain, only one construct was found to influence processes of implementation and this related to knowledge and beliefs about the intervention. Participants emphasised the importance of individual commitment from staff. A strong belief in the relative advantages of ERAS meant that most staff were committed to delivering the service. Resistance to change existed where ERAS practices were seen as being incompatible with professional judgement, as discussed above.

\section{Process}

To plan processes of care, protocols were used to 'streamline' services and ensure patients received key elements of care, although these were not always formally described. However, participants stressed these should be sufficiently flexible to meet individual needs, as discussed above.

A consultant surgeon at the district general hospital (Shinebury) emphasised the importance of sustaining multidisciplinary commitment and advocated 'top-down' encouragement and close monitoring to do so. To facilitate this, staff at the district general hospital (Shinebury) held multidisciplinary meetings to ensure key members of the team were cascading information to colleagues 'to keep that momentum going'. However, a nurse at the specialist orthopaedic hospital (Elmfield) explained that not all team members were invited to meetings to discuss ERAS development and this made them feel less engaged.

Involving strong opinion leaders in the development of ERAS helped to generate internal support whereas a lack of this at the specialist orthopaedic hospital (Elmfield) was a barrier to engagement, as discussed. The importance of having a recognised ERAS champion to 'drive through' changes was highlighted. The teaching hospital (Towerton) had a designated nurse specialist that acted as the central point of contact. As a result, other members of the multidisciplinary team did not need to be familiar with all aspects of the protocol. Similarly, consultants at the district general hospital (Shinebury) were identified as a source of expertise. Clinical champions also helped to engender enthusiasm.

ERAS had to be (re)activated on a continuous basis through reflection, evaluation and modification. To reconfigure care, the staff at the district general hospital (Shinebury) used multidisciplinary meetings to review outcomes data and 'brainstorm' ways of improving services. Informal communication between team members, for instance in hip and knee schools, provided another opportunity for this. Patient feedback was used to shape patient education materials and joint schools. Feedback was collected informally or through patient satisfaction questionnaires. On account of these processes, ERAS was seen as having been improved or 'refined' at three study sites. ERAS at the district general hospital (Shinebury) was described as having a 'core element', which has grown outwards as the service has 'tried to add bits on to try and improve the situation'. By contrast, the staff at the specialist orthopaedic hospital (Elmfield) talked about how ERAS was gradually being 'nibbled at the edges'.

\section{DISCUSSION \\ Overview of findings}

This study used the CFIR to explore how healthcare professionals view ERAS programmes for hip and knee replacement. Findings showed that 17 of the CFIR 31 constructs influenced the implementation of ERAS across all five domains. Within 'intervention characteristics', participants felt ERAS afforded advantages over alternative solutions. Support was higher where ERAS was seen to have been developed internally rather than externally. Guidance was flexible and could be adapted to meet the demands of individual hospital services. In the 'outer setting', participants thought ERAS should be tailored to patient needs and that education could empower them in their recovery. There were concerns about a lack of postdischarge support and tensions between primary and secondary care. In the 'inner setting', one of the key elements of success was effective multidisciplinary collaboration. This was achieved by transferring knowledge about patients along the care pathway, through multidisciplinary team meetings and paperwork. ERAS was a 'message' that had to be communicated to all staff but there were concerns about funding constraints, staffing levels and high volumes of patients. Access to information about the intervention was variable. The characteristics of individuals impacted on implementation and staff were reluctant to change working practices where ERAS was seen as being incompatible with professional judgements. Formal and informal targets were used to inform service delivery. Within 'process', protocols were used to streamline care although these had to be flexible to meet individual needs. Participants thought that 'topdown' encouragement, monitoring and regular meetings helped to ensure team engagement. Involving strong opinion leaders in its development and 'champions' that drove through implementation and acted as a point of contact, helped facilitate implementation. Reviewing outcomes data, informal communication to discuss progress and patient feedback, helped to develop ERAS over time.

\section{How findings relate to current literature}

Findings characterise differences in how ERAS services for hip and knee replacement are delivered by identifying 
barriers and enablers to their successful implementation. This may help to account for variation in health outcomes for these surgeries. ${ }^{11}$ For instance, meeting patient needs may help them to work more successfully towards their own recovery. ${ }^{26}$ Findings reflect those from previous studies that have explored processes that influence implementation of ERAS for other conditions. ${ }^{27-29}$ These found that multidisciplinary collaboration was essential and that this could be threatened by the need to coordinate working practices across different departments. ${ }^{30}$ Likewise, components of ERAS were seen as being incompatible with the working practices of some members of the multidisciplinary team, making staff resistant to change. ${ }^{31}$ A need to engage staff was emphasised and ERAS 'champions' were seen as a means of achieving this goal. ${ }^{29}{ }^{32}$ The importance of providing education to patients and giving them realistic expectations of their recovery was discussed. ${ }^{27}{ }^{32}$ Temporality or strategies to embed ERAS over time were discussed in a small number of studies. ${ }^{28}{ }^{29}$ Studies have been synthesised in a recent systematic review. ${ }^{12}$

Our study contributed to the existing literature by emphasising the importance of meeting patient needs in service design, including the need to ensure that the 'message' of ERAS is successfully and consistently communicated in order to encourage patients to engage in rehabilitative work. It also highlights the need for effective collaboration between primary and secondary care services to provide effective discharge support, reflecting challenges in the wider healthcare system. ${ }^{33}$

Our study highlights the importance of ensuring that protocols are sufficiently flexible to meet individual patient needs. Services should also prioritise strategies to empower patients in their recovery through education. Adequate postdischarge support should be built into services and effective working relationships established with primary care through established channels such as postdischarge documentation. Likewise, multidisciplinary team working around ERAS should be encouraged to ensure that there is a commitment to delivering ERAS, and a consensus about its meaning and how it should be enacted in the service. Education forms an essential component of this. Strong opinion leaders or ERAS 'champions' may be introduced as a source of information for staff and to help engender enthusiasm for the intervention. Establishing formal evaluation processes, along with using informal sources of feedback to help reconfigure services, may be used to ensure that services are refined and delivered over time.

\section{Strengths and weaknesses}

Using ethnographic research methods involved spending extended periods of time at study sites using multiple research methods that provided a rounded account of practice. Analysis included information about what people did as well as what they said, and their reasons for their actions and decisions. ${ }^{34}$ By including 38 healthcare professionals, we aimed to reflect diverse experiences, but the different numbers of participants drawn from each of the study sites meant that experiences at some hospitals could have been over-represented in the analysis. However, this was mitigated by analysing data from each hospital as a discrete data set and then comparing and contrasting findings. On account of this, we think that findings are adequately transferable to other settings. ${ }^{35} \mathrm{In}$ our presentation of findings, we differentiated between individual study sites where relevant. Where findings were similar across all sites, data were pooled. Despite significant variation in service delivery, we found that all constructs were relevant across all study sites.

The CIFR provided a theoretical basis to our analysis. We used CFIR because of its emphasis on meeting patients' needs in service design. Our study highlighted that meeting patients' needs was important to its successful implementation into everyday practice. By using inductive coding and transposing themes onto the theory that we thought was the best 'fit' for the data, we ensured that data were not forced into predefined constructs. A challenge that we encountered in the analysis was how best to make decisions about where themes fitted best, particularly when it was possible that these could be mapped against more than one construct. Where this was the case, themes were mapped onto the construct that was considered to be the best 'fit' or coded into more than one construct.

\section{Further research}

Study participants reflected on the role of primary care in delivering components of ERAS, including processes of referral and postdischarge support. Further research could explore how primary care interacts with ERAS protocols, providing a more comprehensive understanding of the delivery of ERAS. In addition, exploring patients' experiences would provide vital information about how best to meet patient needs. As part of the broader study of which the results described here form a part, we are exploring patients' experiences.

\section{CONCLUSIONS}

ERAS has been heralded as a way of improving care for patients undergoing surgery. Our research demonstrates that successful implementation of ERAS services for hip and knee replacement depends on several aspects, such as the extent to which services have been adapted to meet individual needs, effective communication between staff and planning processes. Doing so provides information to healthcare providers on how best to organise and deliver these services in the future. The study may also be of use to clinicians and researchers in helping to understand service delivery for ERAS in other surgeries.

Acknowledgements We would like to thank members of the PEP-R ('The Patient Experience Partnership in Research') group based at the Musculoskeletal Research Unit, University of Bristol for their input into study design, data collection materials and dissemination strategies. 
Contributors $\mathrm{SD}, \mathrm{AJ}, \mathrm{RC}$ and $\mathrm{RG}-\mathrm{H}$ contributed to study design and data analysis. SD, AJ, RC, RF, KB and RG-H contributed to the interpretation of data and preparation of the manuscript and provided final approval of this version of the manuscript.

Funding This project was funded by the National Institute for Health Research Health Services and Delivery Research Programme (project name ATLAS, project number 14/46/02). Additional support was received from the Oxford NIHR Biomedical Research Centre, Nuffield Orthopaedic Centre, University of Oxford. A and RG-H were supported by the NIHR Biomedical Research Centre at the University Hospitals Bristol NHS Foundation Trust and the University of Bristol.

Disclaimer The views and opinions expressed therein are those of the authors and do not necessarily reflect those of the HS\&DR Programme, NIHR, NHS or the Department of Health.

Competing interests Yes, there are competing interests for one or more authors and I have provided a Competing Interests statement in my manuscript.

Patient consent for publication Not required.

Ethics approval Ethical approval was provided by the South-West Exeter Research Ethics Committee (Ref: 16/SW/0214).

Provenance and peer review Not commissioned; externally peer reviewed.

Data sharing statement At the time this study was performed, participants consented to the data of this study being used for research. Therefore, completely open access of the data would contravene consent and ethics approval. The original study team will have exclusive use of this data for 6 years from the start of the study on 1 April 2016. Data will be kept on the University of Bristol research office's secure server and in hard copy within a secure filing cabinet at the University of Bristol's Musculoskeletal Research Unit. After 1 April 2022, the fully anonymised interviews will be deposited at the University of Bristol Research Data Repository for a further 14 years. Controlled access to the data request must be sought by completing and submitting a request to the University of Bristol Data Access Committee. This will assess the motives of potential researchers before granting access to the dataset. RC will be custodian of the data.

Open access This is an open access article distributed in accordance with the Creative Commons Attribution Non Commercial (CC BY-NC 4.0) license, which permits others to distribute, remix, adapt, build upon this work non-commercially, and license their derivative works on different terms, provided the original work is properly cited, appropriate credit is given, any changes made indicated, and the use is non-commercial. See: http://creativecommons.org/licenses/by-nc/4.0/.

\section{REFERENCES}

1. Surgery and the NHS in numbers. 2017. Available: https://www. rcseng.ac.uk/news-and-events/media-centre/media-backgroundbriefings-and-statistics/surgery-and-the-nhs-in-numbers/

2. Enhanced recovery partnership programme project report. 2011, Department of Health.

3. Larsen K, Hansen TB, Thomsen PB, et al. Cost-effectiveness of accelerated perioperative care and rehabilitation after total hip and knee arthroplasty. J Bone Joint Surg Am 2009;91:761-72.

4. Joint replacement statistics. 2017. Available: http://www.njrcentre. org.uk/njrcentre/Patients/Jointreplacementstatistics/tabid/99/Default. aspx

5. Osteoarthritis: care and management. 2014. Available: nice.org.uk/ guidance/cg177

6. Beswick AD, Wylde V, Gooberman-Hill R, et al. What proportion of patients report long-term pain after total hip or knee replacement for osteoarthritis? A systematic review of prospective studies in unselected patients. BMJ Open 2012;2:e000435.

7. Blom AW, Artz N, Beswick AD, et al. In: Programme Grants for Applied Research. Improving patients' experience and outcome of total joint replacement: the RESTORE programme: NIHR Journals Library, 2016.

8. Malviya A, Martin K, Harper I, et al. Enhanced recovery program for hip and knee replacement reduces death rate. Acta Orthop 2011;82:577-81.

9. Savaridas T, Serrano-Pedraza I, Khan SK, et al. Reduced mediumterm mortality following primary total hip and knee arthroplasty with an enhanced recovery program. A study of 4,500 consecutive procedures. Acta Orthop 2013;84:40-3.
10. Mawdsley MJ, Baker PN, Desai A, et al. Regional uptake an variations in orthopaedic enhanced recovery pathways in knee and hip total arthroplasty. J Perioper Pract 2016;26:118-22.

11. Street A, Gutacker N, Bojke C, et al. Health Services and Delivery Research. In: Variations in outcome and costs among NHS providers for common surgical procedures: econometric analyses of routinely collected data: NIHR Journals Library, 2014.

12. Cohen R, Gooberman-Hill R. Staff experiences of enhanced recovery after surgery: systematic review of qualitative studies. PROSPERO 2017.

13. Improved Clinical Effectiveness through Behavioural Research Group (ICEBeRG). Designing theoretically-informed implementation interventions. Implement Sci 2006;1:4.

14. Consolidated Framework for implementation Research Constructs. [cited 2018 5th March]; Available: https://cfirguide.org/constructs/

15. Damschroder LJ, Lowery JC. Evaluation of a large-scale weight management program using the consolidated framework for implementation research (CFIR). Implement Sci 2013;8:51.

16. Breimaier HE, Heckemann B, Halfens RJ, et al. The Consolidated Framework for Implementation Research (CFIR): a useful theoretical framework for guiding and evaluating a guideline implementation process in a hospital-based nursing practice. BMC Nurs 2015;14:43.

17. Varsi C, Ekstedt M, Gammon D, et al. Using the Consolidated Framework for Implementation Research to Identify Barriers and Facilitators for the Implementation of an Internet-Based PatientProvider Communication Service in Five Settings: A Qualitative Study. J Med Internet Res 2015;17:e262.

18. Reeves S, Kuper A, Hodges BD. Qualitative research methodologies: ethnography. BMJ 2008;337:a1020.

19. Leslie M, Paradis E, Gropper MA, et al. Applying ethnography to the study of context in healthcare quality and safety. BMJ Qual Saf 2014;23:99-105.

20. SAGE Publications I. Purposive Sampling. The SAGE Encyclopedia of Qualitative Research Methods. SAGE Publications, Inc. Thousand Oaks, CA: SAGE Publications, Inc.

21. Lewis-Beck M, Bryman A, Futing Liao T. The SAGE Encyclopedia of Social Science Research Methods, 2004.

22. Braun V, Clarke V. Using thematic analysis in psychology. Qual Res Psychol 2006;3:77-101.

23. Damschroder LJ, Aron DC, Keith RE, et al. Fostering implementation of health services research findings into practice: a consolidated framework for advancing implementation science. Implement Sci 2009;4:50.

24. Tavory. I, Timmermans S. Abductive analysis: theorizing qualitative research. London: The University of Chicago Press, 2014.

25. Gooberman-Hill R, Burston A, Clark E, et al. Involving patients in research: considering good practice. Musculoskeletal Care 2013;11:187-90.

26. Lane JV, Hamilton DF, MacDonald DJ, et al. Factors that shape the patient's hospital experience and satisfaction with lower limb arthroplasty: an exploratory thematic analysis. BMJ Open 2016;6:e010871.

27. Lyon A, Solomon MJ, Harrison JD. A qualitative study assessing the barriers to implementation of enhanced recovery after surgery. World J Surg 2014;38:1374-80.

28. Wagner L, Carlslund AM, Møller C, et al. Patient and staff (doctors and nurses) experiences of abdominal hysterectomy in accelerated recovery programme. A qualitative study. Dan Med Bull 2004;51:418-21.

29. Gotlib Conn L, McKenzie M, Pearsall EA, et al. Successful implementation of an enhanced recovery after surgery programme for elective colorectal surgery: a process evaluation of champions' experiences. Implement Sci 2015;10:99.

30. Alawadi ZM, Leal I, Phatak UR, et al. Facilitators and barriers of implementing enhanced recovery in colorectal surgery at a safety net hospital: a provider and patient perspective. Surgery 2016:159:700-12.

31. Jeff A, Taylor C. Ward nurses' experience of enhanced recovery after surgery: a grounded theory approach. Gastrointestinal Nursing 2014;12:23-31.

32. Pearsall EA, Meghji Z, Pitzul KB, et al. A qualitative study to understand the barriers and enablers in implementing an enhanced recovery after surgery program. Ann Surg 2015;261:92-6.

33. National Collaboration for Integrated Care and Support. Integrated care and support: our shared commitment: London, 2013.

34. Pope C, Mays N. Opening the black box: an encounter in the corridors of health services research. BMJ 1993;306:315-8.

35. Given L. The SAGE Encyclopedia of Qualitative Research Methods, 2008. 